\title{
Control of the Internal Structure of MLO-Based Isasomes by the Addition of Diglycerol Monooleate and Soybean Phosphatidylcholine
}

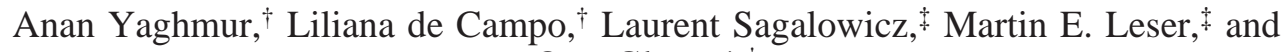 \\ Otto Glatter*,† \\ Institute of Chemistry, University of Graz, A-8010 Graz, Austria, and Nestlé Research Center, \\ Lausanne, Switzerland
}

Received May 9, 2006. In Final Form: August 23, 2006

\begin{abstract}
This work describes the effect of two different surfactants on the internal nanostructure of the kinetically stabilized isasomes (internally self-assembled particles or "somes"), which are a new family of colloidal particles (cubosomes, hexosomes, micellar cubosomes, and emulsified microemulsions, EME). The stabilization of these systems is performed by using the polymeric stabilizer F127. We demonstrate that the internal structure of these oil-free and oil-loaded dispersed particles can be modulated by varying the lipid composition. To achieve this goal, we replaced part of our primary lipid monolinolein (MLO) with diglycerol monooleate (DGMO) or soybean phosphatidylcholine (PC). We found that DGMO has a counter effect to that of tetradecane (TC) and allows us to tune back the self-assembled nanostructure in the TC-loaded dispersions from $\mathrm{H}_{2}$ (hexosomes) to $\mathrm{Im} 3 \mathrm{~m}$ (cubosomes). Although TC has a higher impact on confined structures than does DGMO, we demonstrate that the addition of DGMO significantly affects the internal structure of the TC-solubilized dispersions and favors the formation of large water channels. PC can also be used to modify the internal structure for MLO-based systems. It is somehow different from DGMO due to the fact that the fully hydrated $P n 3 m$ cubic structure in the presence of PC for the TC-free dispersion is preserved after dispersing. The results also indicate that PC is less effective than DGMO for tuning back the TC-loaded internal structure from $\mathrm{H}_{2}$ to cubic phase, in which it makes the confined structure less ordered. In addition, we found that DGMO has a significant effect on the internal structure of isasomes. It increases the water solubilization capacity for dispersed and nondispersed bulk phases. In contrast to the MLO-based dispersions, the present results indicate that F127 plays an important role in the internal structure of these dispersions due to its penetration into the oil-free cubic phase changing the symmetry from $P n 3 m$ to $\operatorname{Im} 3 m$.
\end{abstract}

\section{Introduction}

In the last 20 years, extensive research was conducted on investigating the monoglycerides-based phase diagrams and the factors that determine the specific type of formed assemblies in these rich polymorphic systems. ${ }^{1-9}$ It was found that the structures are affected by the lipid molecular structure (single or double chained, saturation degree, and length of fatty acyl chain), temperature, water content, and the presence of hydrophilic or hydrophobic additives. ${ }^{3-11}$ For example, the well-known binary monoolein (MO)/water system includes a fluid isotropic phase $\left(\mathrm{L}_{2}\right)$, a lamellar $\left(\mathrm{L}_{\alpha}\right)$, an inverted hexagonal $\left(\mathrm{H}_{2}\right)$, and a reversed bicontinuous cubic $\left(\mathrm{V}_{2}\right)$ liquid crystalline phase. ${ }^{1,2,5,12-16}$ It has been shown that the structure of this binary system can be

* Corresponding author. Tel.: +43 316380 5433. Fax: +43 316380 9850. E-mail: otto.glatter@uni-graz.at.

University of Graz

$\doteqdot$ Nestlé Research Center.

(1) Chernik, G. G. Curr. Opin. Colloid Interface Sci. 2000, 4, 381-390.

(2) Larsson, K. Nature 1983, 304, 664

(3) Barauskas, J.; Landh, T. Langmuir 2003, 19, 9562-9565.

(4) Borné, J.; Nylander, T.; Khan, A. Langmuir 2000, 16, 10044-10054.

(5) Qiu, H.; Caffrey, M. Biomaterials 2000, 21, 223-234.

(6) Qiu, H.; Caffrey, M. J. Phys. Chem. B 1998, 102, 4819-4829.

(7) Qiu, H.; Caffrey, M. Chem. Phys. Lipids 1999, 100, 55-79.

(8) Briggs, J.; Chung, H.; Caffrey, M. J. Phys. II France 1996, 6, 723-751.

(9) Misquita, Y.; Caffrey, M. Biophys. J. 2001, 81, 1047-1058.

(10) Caboi, F.; Amico, G. S.; Pitzalis, P.; Monduzzi, M.; Nylander, T.; Larsson, K. Chem. Phys. Lipids 2001, 109, 47-62.

(11) Borné, J.; Nylander, T.; Khan, A. Langmuir 2001, 17, 7742-7751.

(12) Larsson, K. Chem. Phys. Lipids 1972, 9, 181-195. 328 .

(13) Larsson, K.; Fontell, K.; Krog, N. Chem. Phys. Lipids 1980, 27, 321-

(14) Lutton, E. S. J. Am. Oil Chem. Soc. 1965, 42, 1068-1070.

(15) Chung, H.; Caffrey, M. Nature 1994, 368, 224-226.

(16) Hyde, S. T.; Andersson, S.; Ericsson, B.; Larsson, K. Z. Kristallogr. 1984, 168, 213-219. modulated by adding a third component. ${ }^{4,10,11}$ It was found that the nature of the guest molecule significantly influences the phase behavior of this system. ${ }^{10,17,18}$ This effect depends on the amount of the solubilized molecule and on the water content. Molecules leading to the formation of a layer with a positive spontaneous curvature $\left(H_{0}>0\right)$, such as sodium oleate, ${ }^{11}$ induce the transition from $\mathrm{V}_{2}$ to $\mathrm{L}_{\alpha}$, while the addition of an oil, such as oleic acid, induces a structural transition from $\mathrm{V}_{2}$ to $\mathrm{H}_{2}$, that is, leading to the formation of a film layer with negative spontaneous curvature ${ }^{11}$ $\left(H_{0}<0\right)$.

It was reported that the lipid-based structures can be modulated by varying the lipid composition. ${ }^{4,19-21}$ The addition of a nonionic polar lipid ${ }^{19}$ such as diglycerol monooleate (DGMO) to the binary $\mathrm{MO} /$ water system significantly enlarges the lamellar and the cubic phase regions. It was also found that mixing a surfactant favoring the formation of lamellar phases (such as the egg yolk phosphatidylcholine) with a lipid favoring the formation of nonlamellar phases (such as MO or diolein) induces lamellarnonlamellar phase transitions. ${ }^{20,21}$

The submicrometer-sized dispersed particles based on lyotropic liquid crystalline phases of binary surfactant-like lipid/water systems have become of great interest in basic and applied research due to their biological importance, high interfacial area, low

(17) Caboi, F.; Nylander, T.; Razumas, V.; Talaikyte, Z.; Monduzzi, M.; Larsson, K. Langmuir 1997, 13, 5476-5483.

(18) Caboi, F.; Murgia, S.; Monduzzi, M.; Lazzari, P. Langmuir 2002, 18 , 7916-7922.

(19) Pitzalis, P.; Monduzzi, M.; Krog, N.; Larsson, H.; Ljusberg-Wahren, H. Nylander, T. Langmuir 2000, 16, 6358-6365.

(20) Kamo, T.; Nakano, M.; Leesajakul, W.; Sugita, A.; Matsuoka, H.; Handa, T. Langmuir 2003, 19, 9191-9195.

(21) Nakano, M.; Kamo, T.; Sugita, A.; Handa, T. J. Phys. Chem. B 2005, 109 , 4754-4760. 
viscosity, capability to solubilize both hydrophilic and hydrophobic active molecules, various promising applications for the formulation of delivery systems, ${ }^{22-26}$ and for the encapsulation of enzymes and proteins. ${ }^{26}$

In the pioneering work of Larsson and co-workers, ${ }^{26-29}$ it was established that it is possible to form colloidal monoglyceridesbased aqueous dispersions with confined internal nanostructures of $\mathrm{V}_{2}$ (cubosomes) and $\mathrm{H}_{2}$ (hexosomes) by using an efficient stabilizer such as the amphiphilic triblock copolymer Pluronic F127. ${ }^{27-30}$ In recent years, different groups have studied the formation of lipid-based aqueous dispersions and the characterization of their inner periodicity and the outer shape of dispersed soft particles by different techniques: ${ }^{29-39}$ small-angle X-ray scattering (SAXS), cryo-transmission electron microscopy (cryoTEM), atomic force microscopy (AFM), dynamic light scattering (DLS), and ${ }^{13} \mathrm{C}$ NMR. In addition, two novel processes were developed for producing these aqueous dispersions: a dilution process $^{31,40}$ in the presence of ethanol, and an application of microfluidization ${ }^{41}$ followed by heat treatment at $125^{\circ} \mathrm{C}$. Both methods produced submicrometer-sized stable cubosomes. It was also found that the heat treatment at elevated temperatures causes the formation of cubosomes with a narrow size distribution. ${ }^{41}$

There are a number of recent papers, including ours, dealing with the understanding of different structural aspects of the confined nanostructures in the aqueous dispersions by changing temperatures and/or the solubilization of hydrophobic additives, ${ }^{42-45}$ the formation of protein-induced long oriented cubosomic nanoridges ${ }^{46}$ the characterization of the new dispersed

(22) Drummond, C.; Fong, C. Curr. Opin. Colloid Interface Sci. 2000, 4 , 449-456.

(23) Boyd, B. J. Int. J. Pharm. 2003, 260, 239-247.

(24) Siekmann, B.; Bunjes, H.; Koch, M. H. J.; Kirsten, W. Int. J. Pharm. 2002, 244, 33-43.

(25) Spicer, P.; Lynch, M.; Visscher, M.; Hoath, S. In Personal Care Carrier/ Delivery Systems and Formulations; Rosen, M. R., Ed.; Wm Andrew Publishers: New York, 2004.

(26) Ljusberg-Wahren, H.; Nyberg, L.; Larsson, K. Chim. Oggi Chem. Today 1996, 6, 40-43.

(27) Gustafsson, J.; Ljusberg-Wahren, H.; Almgren, M.; Larsson, K. Langmuir 1996, $12,4611-4613$.

(28) Gustafsson, J.; Ljusberg-Wahren, H.; Almgren, M.; Larsson, K. Langmuir 1997, 13, 6964-6971.

(29) Larsson, K. J. Dispersion Sci. Technol. 1999, 20, 27-34.

(30) Larsson, K. Curr. Opin. Colloid Interface Sci. 2000, 4, 449-456.

(31) Spicer, P. T.; Hayden, K. L.; Lynch, M. L.; Ofori-Boateng, A.; Burns, J. L. Langmuir 2001, 17, 5748-5756.

(32) Nakano, M.; Teshigawara, T.; Sugita, A.; Leesajakul, W.; Taniguchi, A.; Kamo, T.; Matsuoka, H.; Handa, T. Langmuir 2002, 18, 9283-9288.

(33) Almgren, M.; Edwards, K.; Karlsson, G. Colloids Surf., A 2000, 174, $3-21$.

(34) Borne, J.; Nylander, T.; Khan, A. J. Phys. Chem. B 2002, 106, 1049210500 .

(35) Spicer, P. T.; Small, W. B.; Lynch, M. L.; Burns, J. L. J. Nanopart. Res. 2002, 4, 297-311.

(36) Nakano, M.; Sugita, A.; Matsuoka, H.; Handa, T. Langmuir 2001, 17, 3917-3922.

(37) Monduzzi, M.; Ljusberg-Wahren, H.; Larsson, K. Langmuir 2000, 16 , $7355-7358$.

(38) Neto, C.; Aloisi, G.; Baglioni, P.; Larsson, K. J. Phys. Chem. B 1999, 103, 3896-3899.

(39) Larsson, K. Curr. Opin. Colloid Interface Sci. 2000, 4, 449-456.

(40) Spicer, P. T. In Encyclopedia of Nanoscience and Nanotechnology; Schwarz, J. A., Contescu, C., Putyera, K., Eds.; Marcel Dekker: New York, 2004; pp 881-892.

(41) Barauskas, J.; Johnsson, M.; Joabsson, F.; Tiberg, F. Langmuir 2005, 21, $2569-2577$.

(42) de Campo, L.; Yaghmur, A.; Sagalowicz, L.; Leser, M. E.; Watzke, H.; Glatter, O. Langmuir 2004, 20, 5254-5261.

(43) Yaghmur, A.; de Campo, L.; Sagalowicz, L.; Leser, M. E.; Glatter, O. Langmuir 2005, 21, 569-577.

(44) Sagalowicz, L.; Michel, M.; Adrian, M.; Frossard, P.; Rouvet, M.; Watzke, H. J.; Yaghmur, A.; de Campo, L.; Glatter, O.; Leser, M. E. J. Microsc. 2006, $221,110-121$.

(45) Yaghmur, A.; de Campo, L.; Salentinig, S.; Sagalowicz, L.; Leser, M. E.; Glatter, O. Langmuir 2006, 22, 517-521.

(46) Angelova, A.; Angelov, B.; Papahadjopoulos-Sternberg, B.; Bourgaux, C.; Couvreur, P. J. Phys. Chem. B 2005, 109, 3089-3093. nanoparticles of the glycolipid bicontinuous cubic phases, ${ }^{47}$ the cubic phases based on mixing MO with PEG-based copolymers bearing lipid-mimetic anchors at certain ratios in glycerol, ${ }^{48}$ and the dispersed phospholipid-based bicontinuous cubic phases ${ }^{49}$ formed when small amounts of PEGylated monoolein are added.

The main goal of the present study is to investigate the effect of varying the lipid composition on the confined internal structures in our dispersed particles. In our previous works, ${ }^{43,45}$ we found that upon increasing the solubilized oil content, there is a significant reduction in the water solubilization capacity in the interior of our aqueous dispersions, ${ }^{43,45}$ and we observed simultaneously a structural transition from $\mathrm{V}_{2} \rightarrow \mathrm{H}_{2} \rightarrow \mathrm{Fd} 3 m \rightarrow \mathrm{W} / \mathrm{O}$ microemulsion (see Figures 1 and 2 in ref 43), that is, leading to the formation of a film layer with more negative spontaneous curvature. We will focus in this study on checking the possibility of changing the lipid composition to have a counter effect to that of oil. This means having an efficient tool to control the oilloaded confined nanostructures. We are interested in keeping the spontaneous curvature of the film layer less negative and in maintaining or even enlarging simultaneously the amount of solubilized water in the oil-loaded interior. Thus, in our present work, the following two main questions will be answered: (a) can the type of the internal structure of the dispersed particles be modulated by replacing part of MLO by DGMO or soybean phosphatidylcholine (PC), and (b) is it possible by modification of the lipid composition to tune back the internal nanostructure from the hexagonal and the W/O microemulsion phases to the bicontinuous cubic phases?

For the investigations described in the present study, we used the monoglyceride monolinolein (MLO) that is less studied in the literature than the well-known monoolein (MO). The MLO enables the formation of all three major phases, that is, the reversed types of the bicontinuous cubic $\left(\mathrm{V}_{2}\right)$, the hexagonal $\left(\mathrm{H}_{2}\right)$, and the microemulsion $(\mathrm{W} / \mathrm{O})$ phase, within a feasible experimental temperature range (between 20 and $95^{\circ} \mathrm{C}$ ). It is also worth noting that we are using commercial lipids; therefore, the structures of the dispersed and nondispersed phases can be affected by the presence of fatty impurities. Thus, in our ongoing investigations, we also focus on the effect of replacing these commercial lipids by highly pure components (approximately 99\%).

The present study is organized in the following way: in the first three parts, the effect of partial replacement of MLO by DGMO on the internal nanostructure of the aqueous dispersions is discussed. Next, the effect of temperature on these confined structures is described, and, finally, we present some results on the effect of partial replacement of MLO by PC on confined nanostructures. It will be shown that the addition of DGMO and PC opens up the possibility to tune back the internal nanostructure in the TC-loaded dispersions from $\mathrm{H}_{2}$ (hexosomes) to $\operatorname{Im} 3 m$ (cubosomes) or modify the self-assembled nanostructure in the dispersed systems.

\section{Materials and Methods}

Materials. Monolinolein (emulsifier TS-PH 039, glycerol monolinoleate (MLO)) and diglycerol monooleate (DGMO) were supplied by Danisco A/S (Brabrand, Denmark). MLO is a distillated monoglyceride that consists of $93.8 \%$ monoglyceride and $4.1 \%$ diglycerides. The fatty acids are composed of $91.8 \%$ linoleate, $6.8 \%$ oleate, and about $1 \%$ saturated fatty acids. Soybean phosphatidylcholine, PC (Epikuron 200), was purchased from Lucas Meyer GMBH Co., Hamburg, Germany. It consists of phosphatidylcholine

(47) Abraham, T.; Hato, M.; Hirai, M. Biotechnol. Prog. 2005, 21, 255-262.

(48) Rangelov, S.; Almgren, M. J. Phys. Chem. B 2005, 109, 3921-3929.

(49) Johnsson, M.; Barauskas, J.; Tiberg, F. J. Am. Chem. Soc. 2005, 127 1076-1077. 
(92\%) and a small amount of accompanying phospholipids (2\%). The distribution of the fatty acids in Epikuron 200 is as reported: saturated fatty acids $=13-17 \%, \mathrm{C}_{18: 1}=6-8 \%, \mathrm{C}_{18: 2}=68-72 \%$, and $\mathrm{C}_{18: 3}=7-9 \%$. Tetradecane (TC) was obtained from the Sigma Chemical Co. (St. Louis, MO). Pluronic F127 ( $\left.\mathrm{PEO}_{99} \mathrm{PPO}_{67}-\mathrm{PEO}_{99}\right)$ was a gift from BASF Corp. (Mount Olive, NJ). All ingredients were used without further purification. The water was double-distilled.

Preparation of Quaternary MLO/DGMO (or PC)/Oil/Water Nondispersed Systems (Bulk Phases). The nondispersed samples were prepared in Pyrex tubes by weighing the appropriate amounts of MLO, DGMO (or PC), oil, and water or aqueous F127 solution, heating them by using an air gun, and homogenizing them by vigorous agitation with a Vortex. They were then left to cool to room temperature and incubated at the same temperature for 1-2 weeks before SAXS measurements were carried out.

Formulation of MLO-Based Oil-Loaded Aqueous Dispersions. A mixture of MLO (viscous liquid), DGMO (or PC), and tetradecane (TC) was weighed into a $20 \mathrm{~mL}$ vial and mixed thoroughly by vortexing. F127 and then water were added to give a sample of a total weight of $10 \mathrm{~g}$. Next, the sample containing all five ingredients was treated by ultrasonication for $20 \mathrm{~min}$, resulting in a milky dispersion. The typical composition of the prepared dispersions was $95 \mathrm{wt} \%$ water and $5 \mathrm{wt} \%$ of a MLO/DGMO (or PC)/oil/F127 mixture, whereby the concentration of the F127 was constant $(0.375$ wt \%). The aqueous dispersions were prepared with various $\alpha$ $(\alpha=(($ mass of TC) $/($ mass of MLO + mass of DGMO (or mass of PC) $\left.)^{*} 100\right)$ and $\beta(\beta=(($ mass of DGMO (or mass of PC) $) /($ mass of MLO) $\left.)^{*} 100\right)$ values. Ultrasonication was carried out using a highintensity ultrasonic processor (SY-LAB G.m.b.H, Pukersdorf, Austria), at $30 \%$ of the maximum power, and $0.5 \mathrm{~s}$ pulses interrupted by $0.5 \mathrm{~s}$ breaks. There was no external sample cooling applied.

Small-Angle X-ray Scattering (SAXS) Measurements. The used SAXS equipment consisted of a SAXSess camera ${ }^{50}$ (Anton-Paar, Graz, Austria), which was connected to an X-ray generator (Philips, PW 1730/10) operating at $40 \mathrm{kV}$ and $50 \mathrm{~mA}$ with a sealed-tube $\mathrm{Cu}$ anode. A Göbel mirror was used to convert the divergent polychromatic X-ray beam into a focused line-shaped beam of $\mathrm{Cu} \mathrm{K}$ radiation $(\lambda=0.154 \mathrm{~nm})$. The $2 \mathrm{D}$ scattering pattern was recorded by an imaging-plate detector (model Fuji BAS1800 from Raytest, Straubenhardt, Germany) and integrated into a one-dimensional scattering function $I(q)$ using SAXSQuant software (Anton Paar, Graz, Austria), where $q$ is the length of the scattering vector, defined by $q=(4 \pi / \lambda) \sin \theta / 2, \lambda$ being the wavelength and $\theta$ the scattering angle. For indexing the different mesophases, we used the reflection laws summarized in the Supporting Information of our recent work. ${ }^{42}$ These comprise the space groups that have been determined for the cubic and the hexagonal phases containing membrane lipids. ${ }^{51}$ The interplanar distance $d$ between two reflecting planes is given by $d=2 \pi / q$, which enables us to calculate the corresponding mean lattice parameter $a$. The scattering profiles of the so-called $\mathrm{L}_{2}$ phase show only one broad correlation peak, for which the position of the observed maximum is shifted to lower $q$ values due to the "smearing effects" of the line-shaped primary beam. These profiles were therefore desmeared by fitting these data with the generalized indirect Fourier transformation (GIFT) method. ${ }^{52}$ For the $\mathrm{L}_{2}$ phase, $d$ is called a characteristic distance.

The sample was filled at room temperature into the sample holder (capillary in a metal block, temperature controlled by a Peltier element, $\pm 0.1^{\circ} \mathrm{C}$ ) and equilibrated at each experimental temperature for at least $10 \mathrm{~min}$ before measurement. All temperature scans were performed in heating direction. An exposure time of 10 and $45 \mathrm{~min}$ was taken for the nondispersed and dispersed samples, respectively.

Cryogenic Transmission Electron Microscopy (Cryo-TEM). A laboratory-built controlled environment vitrification system for Cryo-TEM (very similar to the one described by Egelhaaf et al. ${ }^{53}$ ) was used. Humidity in the environment chamber was about $95 \%$ for

(50) Bergmann, A.; Orthaber, D.; Scherf, G.; Glatter, O. J. Appl. Crystallogr. 2000, 33, 869-875.

(51) Lindblom, G.; Rilfors, L. Biochim. Biophys. Acta 1989, 998, 221-256. 1216

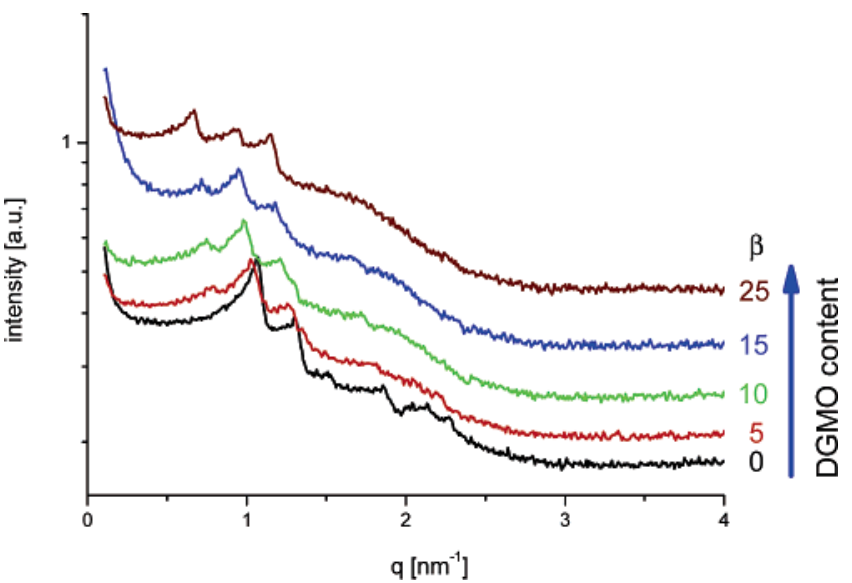

Figure 1. Effect of diglycerol monooleate (DGMO) content on the scattering curves of MLO-based aqueous dispersions at $25^{\circ} \mathrm{C}$ for $\alpha$ ratio $=0$ and with different $\beta$ values in the range of $0-25$. The intensities were shifted by a constant arbitrary factor for better visibility.

cubosomes and $100 \%$ for the other dispersions. The environmental chamber temperature was maintained at $25{ }^{\circ} \mathrm{C}$. A droplet of $5 \mu \mathrm{L}$ of dispersion was deposited onto a 200 mesh copper grid $\left(25^{\circ} \mathrm{C}\right.$, Quantifoil R2/2 or S7/2; both Jena, Germany) that was covered with a carbon film containing holes. It was left between two filter papers 595 (Schleicher \& Schuell, Dassel, Germany) for about 2 s before being propelled into liquid ethane. Frozen grids were stored in liquid nitrogen and transferred into a Cryo-holder (Gatan 6556, Pleasanton, USA) that was kept at $-180^{\circ} \mathrm{C}$. Sample analysis was performed in a Philips CM12 TEM (Philips, Eindhoven, Netherlands) at a voltage of $80 \mathrm{kV}$. Low dose procedures were applied to minimize beam damage. The images were recorded with a slow scan digital camera Gatan 694 (Pleasanton, USA).

\section{Results and Discussion}

\section{Effect of Diglycerol Monooleate (DGMO) on the Internal} Structure of MLO-Based Aqueous Dispersions. Figure 1 shows the effect of DGMO on the internal structure of the MLO-based aqueous dispersions, stabilized by F127. The SAXS scattering curves with five different $\beta$ values (in the range of $0-25$ ) at 25 ${ }^{\circ} \mathrm{C}$ are shown: in the absence of oil and DGMO (at $\alpha=0$ and $\beta=0$ ), the scattering curve of the dispersion shows six peaks in the characteristic ratio for a cubic structure of the $C_{D}$ (diamond)type $P n 3 m$ (cubosomes, emulsified cubic phase ECP). At low DGMO concentrations (at $\beta=5$ and 10), the scattering curves still show the characteristic peaks for a cubic $P n 3 m$ structure, and an additional peak at low $q$ values. At a higher DGMO concentration, the observed three peaks are in the characteristic ratio for a cubic phase of the symmetry $\operatorname{Im} 3 m$; an exemplary scattering curve is shown in Figure 1 for $\beta=25$ (the highest $\beta$ value used in this study). The appearance of the additional peak at low DGMO content suggests the formation of two phases within the confined internal nanostructures of our dispersed particles: the $P n 3 m$ cubic phase (bicontinuous, diamond type $\mathrm{C}_{\mathrm{D}}$ ) coexists with the Im $3 m$ cubic phase (bicontinuous, primitive type $\mathrm{C}_{\mathrm{P}}$ ). This result is confirmed by previous investigations on the internal nanostructure of MO-based cubosomes that were stabilized by F127. ${ }^{32,36}$ Recently, the coexistence of $C_{D}$ and $C_{P}$ phases was also observed in the confined structure of the polymer dispersed bicontinuous glycolipid phases. ${ }^{47}$ The addition of DGMO shifts the peaks to lower $q$ values and increases the structure parameters (see Table 1). This significant change in the

(53) Egelhaaf, S. U.; Schurtenberger, P.; Müller, M. J. Microsc. 2000, 200 $128-139$. 
Table 1. Structure Parameters (Mean Lattice Parameter, $a$ ) for the Dispersed Cubic Phases As Derived from SAXS

Investigations That Were Carried Out at $25^{\circ} \mathrm{C}$ on the Oil-Free

Dispersed MLO-Based Systems $(\alpha=0)$ as a Function of $\beta^{a}$

\begin{tabular}{rlllc}
\hline \multicolumn{1}{c}{$\begin{array}{c}\text { space } \\
\text { group }\end{array}$} & $\begin{array}{c}a_{P n 3 m} \\
{[\mathrm{~nm}]}\end{array}$ & $\begin{array}{c}a_{I m 3 m} \\
{[\mathrm{~nm}]}\end{array}$ & $\begin{array}{c}\text { Bonnet ratio: } \\
a_{I m 3 m} / a_{P n 3 m}\end{array}$ \\
\hline 0 & $P n 3 m$ & 8.6 & & \\
5 & $P n 3 m$ and $\operatorname{Im} 3 m$ & 8.71 & 11.36 & 1.30 \\
10 & $P n 3 m$ and $\operatorname{Im} 3 m$ & 8.98 & 11.91 & 1.33 \\
15 & $P n 3 m$ and $\operatorname{Im} 3 m$ & 9.25 & 12.37 & 1.34 \\
25 & $\operatorname{Im} 3 m$ & & 13.37 &
\end{tabular}

${ }^{a}$ The aqueous dispersions contain $95 \mathrm{wt} \%$ water and $5 \mathrm{wt} \%$ of a mixture of MLO, DGMO, and F127, whereby the concentration of the F127 was constant $(0.375$ wt \%).

mean lattice parameter, $a$, for the dispersed $P n 3 m$ and $\operatorname{Im} 3 m$ cubic phases reveals structural modifications due to the presence of DGMO. Under the assumption that the spontaneous curvature model remains unchanged for bicontinuous cubic phases during the phase transition from one bicontinuous symmetry to another, the Bonnet ratio, given in eq 1 , predicts the respective ratios of the lattice parameters of two different cubic phases with periodic minimal surface (PMS) structures. ${ }^{54}$

$$
\frac{a_{\operatorname{Im} 3 m}}{a_{P n 3 m}}
$$

$a_{I m 3 m}$ and $a_{P n 3 m}$ denote the lattice parameters for the $P n 3 m$ and Im $3 m$ cubic phases, respectively. As shown in Table 1 for our dispersed particle with confined $P n 3 m$ and $\operatorname{Im} 3 m$ structures, the Bonnet ratios are in the range of $1.3-1.34$. These values are in good agreement with the theoretical value ${ }^{54}$ of 1.279 and with the reported ratio of 1.33 for the glycolipid-dispersed particles with confined $P n 3 m-\operatorname{Im} 3 m$ coexisting phases. ${ }^{47}$ Further examples of lipid/water systems that are consistent with the Bonnet model are given in the recent review of Larsson and Tiberg. ${ }^{55}$

To better characterize the effect of DGMO on the internal nanostructures of the MLO-based aqueous dispersions, the corresponding nondispersed bulk systems (mesophases coexisting with excess water) were also investigated. This allows a direct comparison of the confined nanoscaled internal structure of the aqueous dispersions with those of the corresponding nondispersed bulk systems and, as a consequence, a better understanding of the observed structural changes in the dispersed systems.

It is worth noting that the dispersed internal structures, as well as the structures of the nondispersed bulk samples, depend not only on the DGMO content ( $\beta$ value), but of course also on the water content in the respective phase. Figure 2 shows how the structures of MLO/DGMO mixtures at $\beta=25$ change with increasing water content. At a low water content, a fluid isotropic phase with no long-range order (so-called $\mathrm{L}_{2}$ ) is formed (the scattering curves of this system have a single broad peak), and at a higher water content (10 and 15 wt $\%$ water), an $\mathrm{L}_{\alpha}$ phase is formed. Thereby, with increasing water content in the single phase regions, the observed peaks move to lower angles, corresponding to larger structures ("swelling" with water). At 20 wt $\%$ water, a cubic structure of the type Ia3d is formed. The mean lattice parameter for this phase significantly increases from 11.55 to $16.64 \mathrm{~nm}$ as the water content in the monophasic samples changes in the range of $20-40 \mathrm{wt} \%$. From a comparison with the binary MLO/water system as shown in Table 2, it is obvious that the inclusion of DGMO at $25^{\circ} \mathrm{C}$ significantly increases the

(54) Hyde, S. T. Curr. Opin. Solid State Mater. Sci. 1996, 1, 653-662. 369.

(55) Larsson, K.; Tiberg, F. Curr. Opin. Colloid Interface Sci. 2005, 9, 365-
Table 2. Structure Parameters (Mean Lattice Parameter, a) for the Nondispersed Cubic Phases As Derived from SAXS

Investigations That Were Carried Out at $25^{\circ} \mathrm{C}$ on the Oil-Free Dispersed MLO-Based Systems $(\alpha=0)$ for the Binary MLO-Water $(\beta=0)$ and the Ternary MLO-DGMO-Water $(\beta=25)$ Systems

\begin{tabular}{|c|c|c|c|}
\hline $\begin{array}{c}\text { investigated } \\
\text { nondispersed system }\end{array}$ & $\begin{array}{l}\text { water } \\
\text { content }\end{array}$ & $a[\mathrm{~nm}]$ & $\begin{array}{l}\text { space } \\
\text { group }\end{array}$ \\
\hline \multirow{8}{*}{ MLO-water } & 5 & 3.67 & $\mathrm{~L}_{2}$ \\
\hline & 10 & 9.44 & $\operatorname{Ia} 3 d$ \\
\hline & 15 & 10.07 & $I a 3 d$ \\
\hline & 20 & 10.74 & $\operatorname{Ia3d}$ \\
\hline & 25 & 12.36 & $I a 3 d$ \\
\hline & 30 & 8.28 & $P n 3 m$ \\
\hline & 35 & 8.30 & $P n 3 m$ \\
\hline & $40^{a}$ & 8.39 & Pn3m \\
\hline \multirow[t]{9}{*}{ MLO-DGMO-water $(\beta=25)$} & 5 & 3.67 & $\mathrm{~L}_{2}$ \\
\hline & 10 & 3.80 & $\mathrm{~L}_{\alpha}$ \\
\hline & 15 & 4.21 & $\mathrm{~L}_{\alpha}$ \\
\hline & 20 & 11.55 & $\operatorname{Ia3d}$ \\
\hline & 25 & 12.65 & $\operatorname{Ia} 3 d$ \\
\hline & 30 & 13.30 & $I a 3 d$ \\
\hline & 35 & 15.16 & $\operatorname{Ia} 3 d$ \\
\hline & 40 & 16.64 & $I a 3 d$ \\
\hline & $\mathbf{5 0}^{a}$ & 10.66 & Pn3m \\
\hline \multirow[t]{6}{*}{ MLO-PC-water $(\beta=25)$} & 5 & 3.42 & $\mathrm{~L}_{2}$ \\
\hline & 10 & 4.41 & $\mathrm{H}_{2}$ \\
\hline & 15 & 4.50 & $\mathrm{H}_{2}$ \\
\hline & 20 & 12.03 & $\operatorname{Ia} 3 d$ \\
\hline & 30 & 12.19 & $I a 3 d$ \\
\hline & 40 & 9.47 & $P n 3 m$ \\
\hline
\end{tabular}

${ }^{a}$ These samples represent SAXS data for the fully hydrated nondispersed mesophases coexisting with excess water.

maximum solubilized amount of water in the bicontinuous cubic phases from approximately $32 \mathrm{wt} \%{ }^{42}$ to approximately $41 \mathrm{wt}$ $\%$ water at $25^{\circ} \mathrm{C}$. The addition of DGMO is well suited for increasing the lattice parameters of dispersed and nondispersed MLO-based cubic phases (see Tables 1 and 2). With a further increase in the water content (at approximately $42 \mathrm{wt} \%$ water), the system exhibits a two-phase region of coexisting Ia $3 d$ and $P n 3 m$ structures, and at approximately 44 wt \% a complete transition from $I a 3 d$ to the fully hydrated $P n 3 m$ phase takes place. The ratio of the mean lattice parameter of $\operatorname{Ia3d}\left(a_{I a 3 d}\right)$ to that of the $P n 3 m\left(a_{P n 3 m}\right)$ is about 1.61, which is again in good agreement with the Bonnet ratio between these two phases (the theoretical ratio is $1.576^{54}$ ), assuming that $I a 3 d$ and $P n 3 m$ have the same curvature of the bilayer in coexisiting phases at the transition. ${ }^{54,55}$ In addition, there is no change in the lattice parameter of the $P n 3 m$ phase beyond the water solubilization limit (at approximately $41 \mathrm{wt} \%$ ).

A simple approach for the prediction of the tendency of a particular lipid (or surfactant) to form mesophases (such as micellar solutions, hexagonal, cubic phases) is described in the literature. 56 The approach takes into account an "effective" molecular geometry of the lipids. This geometric effect is described by the well-known critical packing parameter $(\mathrm{CPP})=$ $v_{\mathrm{s}} / a_{0} l$, where $v_{\mathrm{s}}$ is the hydrophobic chain volume, $a_{0}$ is the headgroup area, and $l$ is the hydrophobic chain length. ${ }^{57}$ Inverted type mesophases are favored if the "effective" CPP is increased. Concerning the systems discussed in the present work, the addition of DGMO leads to an increase in the average headgroup area due to a second glycerol group, and the average hydrophobic chains are less bulky due to the presence of only one double bond in DGMO instead of two double bonds in MLO, which suggests

(56) Larsson, K. J. Phys. Chem. 1989, 93, 7304-7314.

(57) Isrealachvilli, J. N.; Mitchell, D. J.; Ninham, B. W. J. Chem. Soc., Faraday Trans. 2 1976, 72, 1525-1568. 


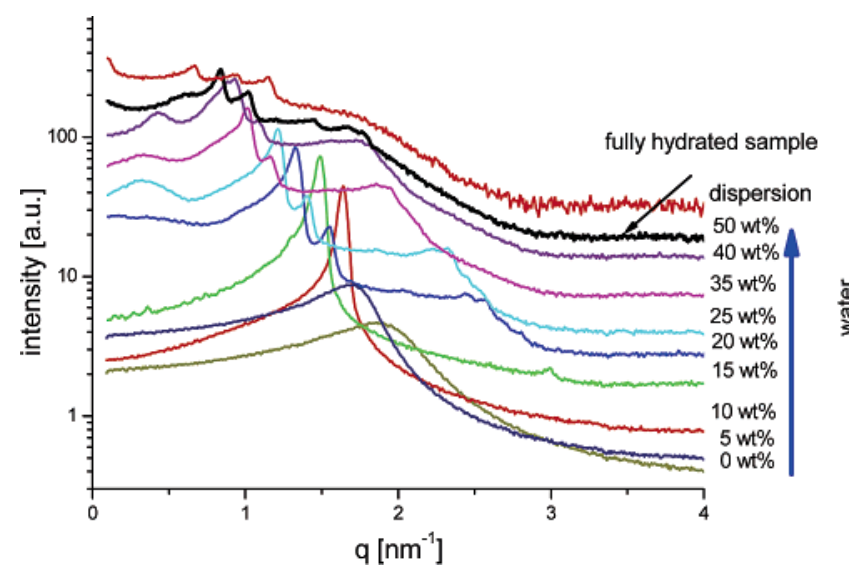

Figure 2. Comparison of the scattering curve from the oil-free MLO-based dispersion $(\alpha=0, \beta=25$, red line) with those from the oil-free nondispersed MLO/DGMO bulk samples at $\beta=25$ with different water content at $25^{\circ} \mathrm{C}$. The intensities were shifted by a constant arbitrary factor for better visibility. The thick black line, in this figure and in the following figures where it is shown, denotes the scattering curve of the fully hydrated bulk sample.

a decrease of the packing parameter and makes the enlargement of the monophasic $\mathrm{L}_{\alpha}$ and Ia $3 d$ cubic regions plausible. ${ }^{19}$ It is well known that DGMO favors the formation of $\mathrm{L}_{\alpha}$, which implies that the value of $\mathrm{CPP} \approx 1 .{ }^{19,58,59}$ Therefore, it is logical that the DGMO/MLO mixture favors the Ia3d phase that borders the $\mathrm{L}_{\alpha}$ in the binary MLO/water system. It was reported that the transformation from the Ia3d surface structure to the $P n 3 m$ surface in $\mathrm{MO}$-water at room temperature increases the CPP from 1.27 to $1.31{ }^{56}$ It was proposed that the effect of the addition of surface active molecules, which are termed "anchors" to surfactant-like lipids on the structure of the liquid crystalline phases, is related to their "locations". $40,55,60$

Recently, we found that the scattering curves of the MLObased dispersions correspond to those of the nondispersed fully hydrated bulk phases. ${ }^{42,43}$ However, as shown for a typical example of these dispersions in Figure 2, upon addition of DGMO there is a disagreement between the structural parameters measured in the dispersed particles and the nondispersed bulk (mesophases coexisting with excess water) systems at the same $\beta$ values. The addition of DGMO promotes the formation of a fully hydrated $P n 3 m$ cubic phase in bulk, while the dispersed particles have an internal structure of the type $\operatorname{Im} 3 \mathrm{~m}$. This means that we have to take into account a possible role of the stabilizer F127 on the internal structure after the dispersing process, as discussed below.

In Figure 3, cryo-TEM images of the sample corresponding to $\alpha=25$ are shown. As always reported, cubosomes are observed to be attached to vesicles (Figure 3a). Crystallographic analysis of the cryo-TEM images confirms that of SAXS. In Figure 3b,c, the electron beam is aligned with the [100] direction of the particle and the $\{110\}$ and $\{200\}$ planes contribute to produce a square motif. In Figure 3d,e, the electron beam is oriented along [111] and a hexagonal motif, formed by the $\{110\}$ planes, is observed. As described in our previous work, ${ }^{44}$ tilting of the particle was performed to discriminate between the $\operatorname{Im} 3 m$ and $P n 3 m$ space groups. Tilting by approximately $30^{\circ}$ enables access to the [110] axis of the observation where the $\{110\},\{200\}$, and $\{211\}$

(58) Holstborg, J.; Pederson, B. V.; Krog, N.; Olesen, S. K. Colloids Surf., B 1999, 12, 383-390.

(59) Johnsson, M.; Lam, Y.; Barauskas, J.; Tiberg, F. Langmuir 2005, 21, 5159-5165.

(60) Lynch, M. L.; Ofori-Boateng, A.; Hippe, A.; Kochvar, K.; Spicer, P. T. J. Colloid Interface Sci. 2003, 260, 404-413. reflections contribute (Figure 3f,g). More important, the $\{1 \overline{1} 1\}$ and the $\{1 \overline{1} \overline{1}\}$ are absent, showing that the space group is $\operatorname{Im} 3 m$ and not Pn3m. ${ }^{44}$ The lattice parameter was found to be approximately $14 \mathrm{~nm}$, which is in relatively good agreement with the SAXS analysis. In this sample, we also observe a few particles with a lattice parameter in the range of $10-11 \mathrm{~nm}$, which are likely to have a space group $P n 3 m$. The presence of a low number of small particles with a different internal structure might not have been detected by SAXS.

2. Effect of the Stabilizer on the Internal Structure of MLO/ DGMO-Based Aqueous Dispersions. Because of the disagreement between the structures in the dispersed MLO/DGMO particles (cubic $\operatorname{Im} 3 \mathrm{~m}$ ) and the fully hydrated nondispersed bulk phase (cubic $P n 3 m$ ), we decided to study the effect of the polymer in the bulk phase. We investigated the structures formed in the nondispersed bulk system of DGMO/MLO mixtures (at $\beta$ value of 25) with $40 \mathrm{wt} \%$ aqueous solution that contain different amounts of F127 in the range of $0-2 \mathrm{wt} \%$. For reasons of comparison, the fully hydrated $P n 3 m$ phase is also shown. In the absence of F127, as presented in Figure 4 and in our results above, an isotropic cubic phase of the symmetry Ia3d is formed. When the added aqueous phase contains F127, a structural transition takes place. The measured peaks of such samples are rather few and very broad; in addition, these samples take a very long time to equilibrate (weeks). However, it can be clearly seen in Figure 4 that at $1 \mathrm{wt} \% \mathrm{~F} 127$, three peaks in the characteristic ratio of cubic $\operatorname{Im} 3 \mathrm{~m}$ are present. A further increase of the polymer concentration up to $2 \mathrm{wt} \% \mathrm{~F} 127$ forms a heterogeneous system; the bulk Im3m cubic phase coexists with excess water plus F127. This means that the polymer incorporates into the bulk of the cubic phase matrix. Landh ${ }^{61}$ studied the phase diagram of the ternary MO/water/F127 system in detail. He found that the cubic phase extends through a wide range of water and polymer contents.

As shown in Figure 4, the comparison of the scattering curves of these bulk nondispersed samples to that of the aqueous dispersion with the same $\beta$ value indicates that part of F127 is inserted into the confined interior of the dispersed particles changing the symmetry of the cubic phase without destroying its structure, while the other part does its job as stabilizer by adhering to the surface of the dispersed particles. This result is different from our recent findings on the effect of F127 with the same concentrations on the symmetry of the dispersed phases in the binary MLO/water and the ternary MLO/TC/water systems. ${ }^{42,43}$ It is there that we found that F127 is an efficient stabilizer and its addition does not affect the symmetry of the dispersed phases. A possible explanation for this difference might simply be the size of the hydrophilic channels in these systems: the hydrophilic channels in the binary MLO/water system are smaller than those in the MLO/DGMO/water or $\mathrm{MO} /$ water system, which might be the reason the polymer cannot be incorporated as easily in the former case. It is also worth noting that when we studied the effect of F127 on the phase behavior of the binary bulk MLO/water system, we found that it is difficult to accommodate the stabilizer's molecules in the monophasic cubic phase region, and, therefore, already a small amount of F127 leads to biphasic samples ( $P n 3 m$ cubic phase coexists with polymer-rich water). As opposed to that, Landh $^{61}$ and Nakano ${ }^{32}$ reported on the accommodation of high F127 concentrations (more than $30 \mathrm{wt} \%$ polymer to lipid ${ }^{61}$ ) in the monophasic MObased cubic phase region, which induces transitions between different cubic phases.

It should be mentioned that the structure of the aqueous dispersions of $\mathrm{MO} /$ water system is influenced by the concentration

(61) Landh, T. J. Phys. Chem. 1994, 98, 8453-8467. 

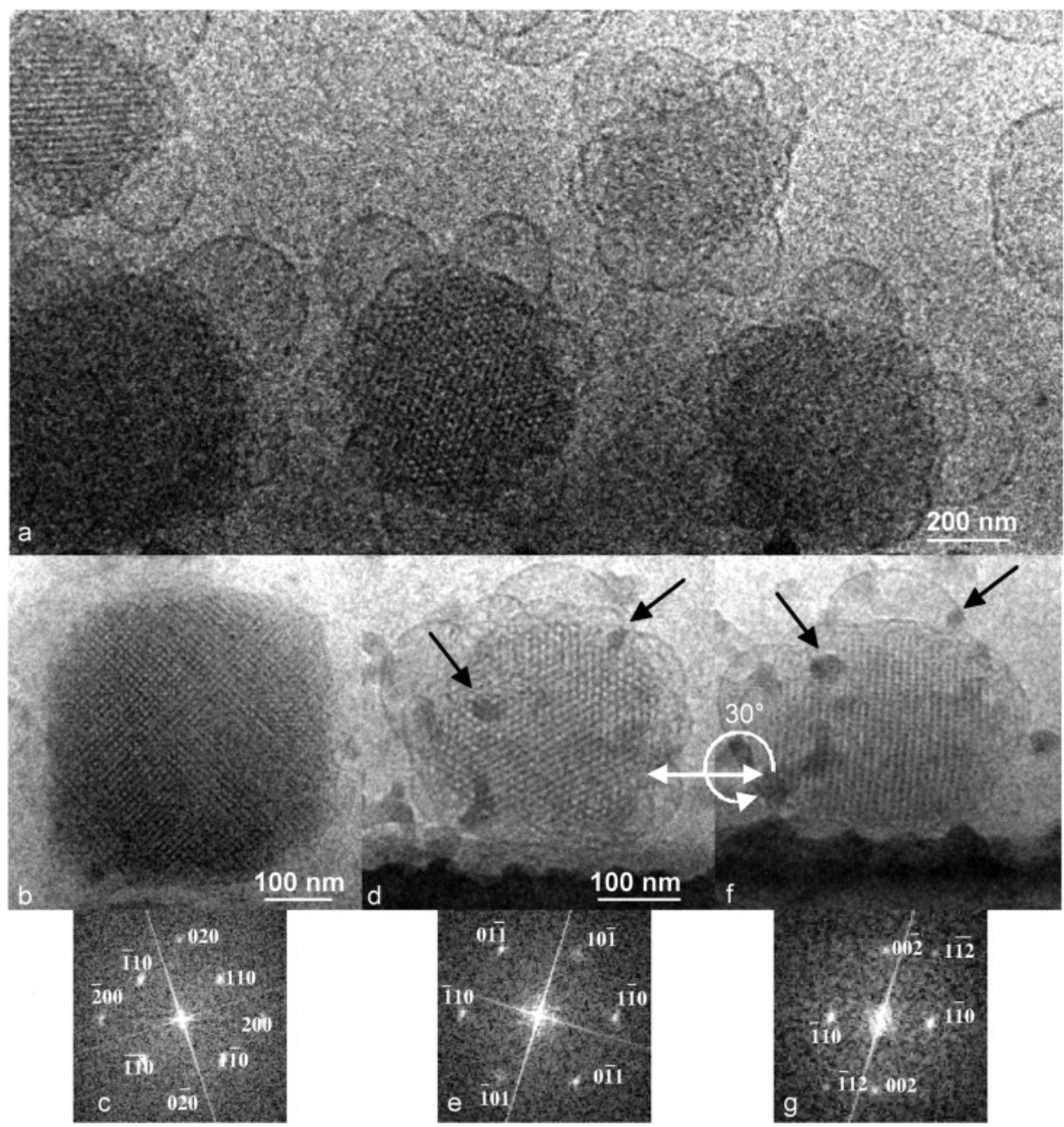

Figure 3. Cryo-TEM images of the oil-free cubosomes based on MLO/DGMO obtained $(\alpha=0, \beta=25)$. (a) Image of several cubosomes, (b) image of a cubosome when the electron beam is parallel to [100], (c) FFT corresponding to (b). (d) The electron beam is oriented along the [111] direction. (e) FFT corresponding to (d). (f) Same particle as in (d) but after tilting the particle by $30^{\circ}$ such that the electron beam is parallel to [110]. (g) Fourier transform of (f). Note that the $\{002\}$ reflection is present while the (111) and the (11 $\overline{1})$ are absent, showing that the space group is $\operatorname{Im} 3 \mathrm{~m}$ and not $P n 3 m$. Arrows in (d) and (f) indicate features that are likely to come from surface contamination. (d) and $(\mathrm{g})$ are reprinted from ref 44.

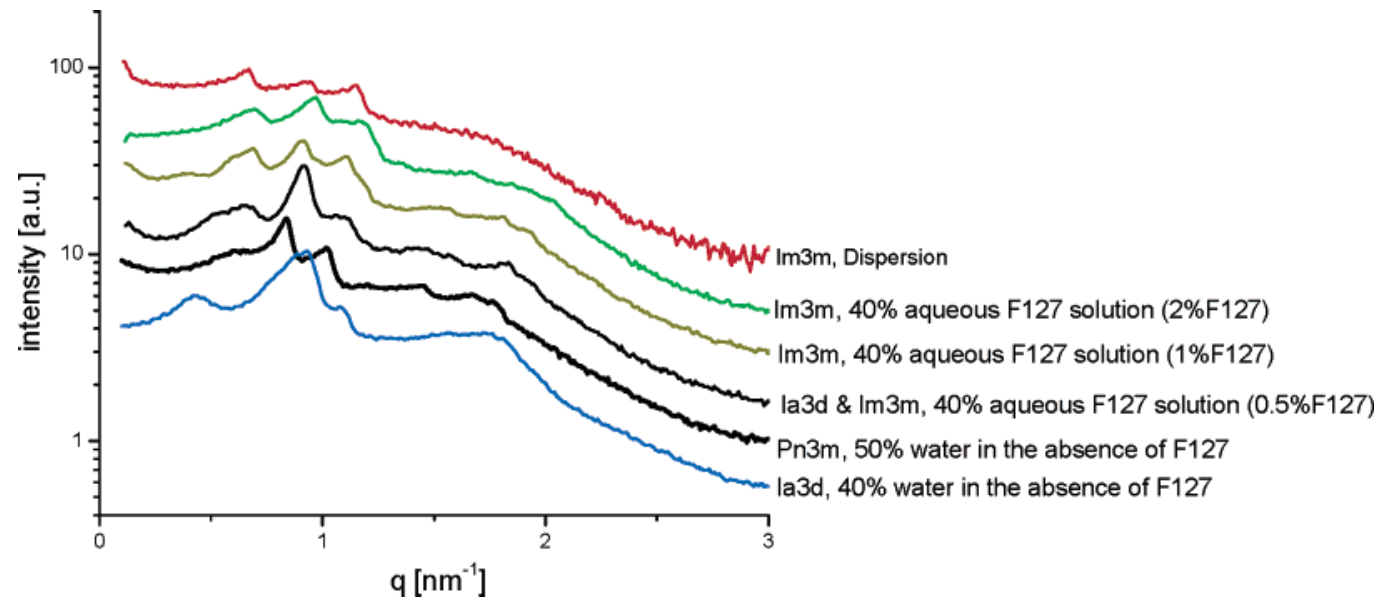

Figure 4. Comparison of the scattering curve from the oil-free MLO-based dispersion $(\alpha=0, \beta=25$, red line) with that from the oil-free nondispersed MLO/DGMO bulk sample at $\beta=25$ that contains $40 \mathrm{wt} \% \mathrm{~F} 127$ aqueous solution. The effect of the stabilizer F127 concentration on this nondispersed cubic phase was carried out at $25^{\circ} \mathrm{C}$ with aqueous solutions containing F127 in the range of $0-2$ wt $\%$. The curves are shifted by a constant arbitrary factor for better visibility.

of F127. Nakano et al. ${ }^{32}$ and Gustafsson et al. ${ }^{28}$ found that at low F127 concentration most of the polymer adsorbs onto the outer surface of the particles and therefore the internal $P n 3 m$ phase is preserved. While at F127 concentrations comparable to those in our investigations, the internal cubic phase transforms from a symmetry of the type $P n 3 m$ to a symmetry of $\operatorname{Im} 3 m$ as a result of the polymer partition into the cubic phase.

3. Effect of Diglycerol Monooleate (DGMO) on the Internal Structure of Oil-Loaded MLO-Based Aqueous Dispersions. To check the possibility of having a counter effect to that of TC, 


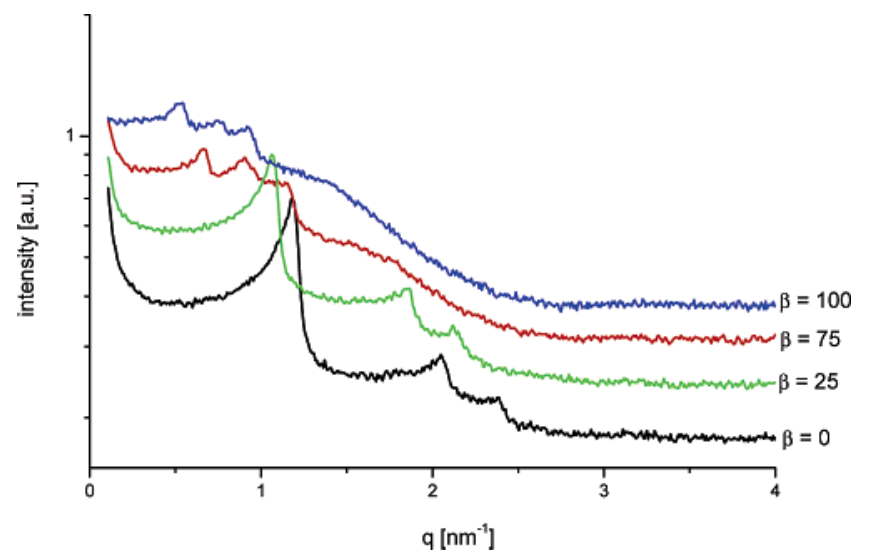

Figure 5. Effect of DGMO content on the scattering curves of TC-loaded aqueous dispersions at $25^{\circ} \mathrm{C}$ for $\alpha=6$, and with different $\beta$ values in the range of $0-100$. The intensities were shifted by a constant arbitrary factor for better visibility.

Table 3. Structure Parameters (Mean Lattice Parameter, $a$ ) for the Dispersed Phases As Derived from SAXS Investigations

That Were Carried Out at $25^{\circ} \mathrm{C}$ on the TC-Loaded Dispersed MLO-Based Systems $(\alpha=6)$ as a Function of $\beta^{a}$

\begin{tabular}{rllr}
\hline \multicolumn{1}{c}{$\beta$} & $\alpha$ & space & $\begin{array}{c}a \\
{[\mathrm{~nm}]}\end{array}$ \\
\hline 0 & 6 & group & 6.13 \\
25 & 6 & $\mathrm{H}_{2}$ & 6.83 \\
50 & 6 & $\mathrm{H}_{2}$ & 13.39 \\
100 & 6 & $\operatorname{Im} 3 m$ & 16.69
\end{tabular}

${ }^{a}$ The aqueous dispersions contain $95 \mathrm{wt} \%$ water and $5 \mathrm{wt} \%$ of a mixture of MLO, DGMO, TC, and F127, whereby the concentration of the F127 was constant (0.375 wt \%).

we investigated the effect of DGMO on the structure of the $\mathrm{MLO} / \mathrm{TC} /$ water system in its colloidal emulsified state; MLO/ DGMO/TC mixtures with different $\beta$ values (in the range of $0-100$ ) and a fixed $\alpha$ value of 6 were dispersed in large amounts of excess water using the block copolymer F127 as stabilizer. The SAXS scattering curves with different $\beta$ values (in the range of $0-100$ ) at $25^{\circ} \mathrm{C}$ are shown in Figure 5: in the absence of DGMO (MLO-based, oil loaded dispersion), the scattering curve of the dispersion shows three peaks in the characteristic ratio for a hexagonal phase (hexosomes). When part of MLO is replaced by DGMO (at $\beta=25$ ), the observed three peaks in the scattering curve move to lower angles, corresponding to larger structures (see Table 3). A further increase of the DGMO concentration in the dispersion ( $\beta=50$ and 100) leads to a scattering curve, which shows the characteristic ratio for a cubic structure of the type Im $3 m$ (cubosomes). Previously, we found that the replacement of MLO by TC induces a transition from cubosomes via hexosomes to EME. ${ }^{43}$ However, as shown in Figure 5, DGMO promotes at $\alpha=6$ a reversed transition (from hexosomes at $\beta=0$ and 25 back to cubosomes at $\beta=50$ and 100). This means that this surfactant has a counter effect on the internal confined structure of the particles to that of the oil (TC). So, DGMO tunes back the structure and makes the spontaneous curvature of the oil-loaded interior less negative. It is worth noting that a higher amount of surfactant $(\beta=50)$ than oil is needed to overcome the effect of TC, which indicates that TC has a higher impact on the internal structure than DGMO does. Despite the fact that at a higher solubilized TC content (at $\alpha=19$ and 75 as presented in Figure 6), the replacement of a relatively high amount of MLO by DGMO $(\beta=100$ (for $\alpha=19$ and 75)) significantly shifts the peaks in the scattering curves to lower angles, corresponding to larger structures, our results reveal that DGMO is not efficient enough to tune back the internal structure from

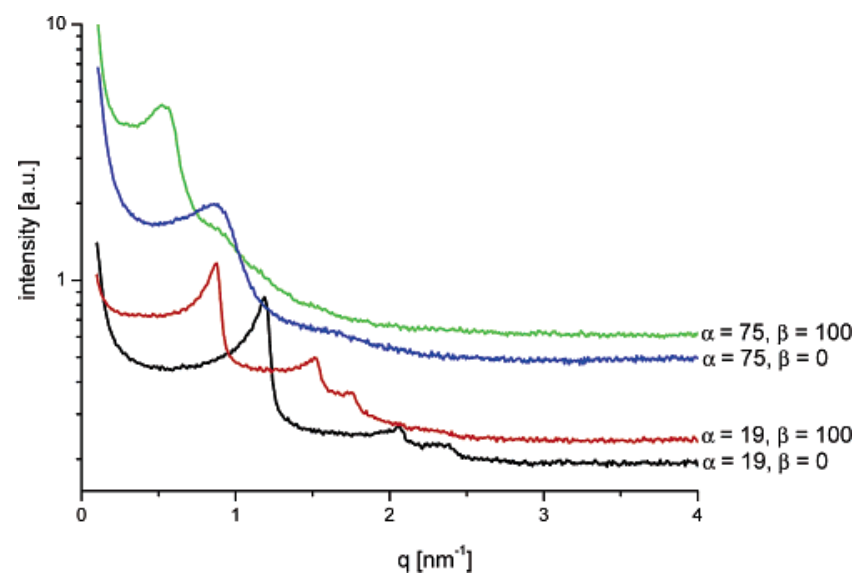

Figure 6. Effect of DGMO content on the scattering curves of TC-loaded aqueous MLO-based dispersions at $25^{\circ} \mathrm{C}$ having $\alpha$ ratios of 19 and 75 , respectively. The scattering curves of the DGMO-free dispersions $(\beta=0)$ are compared to those containing DGMO $(\beta=$ $100)$. The intensities were shifted by a constant arbitrary factor for better visibility.

Table 4. Structure Parameters (Mean Lattice Parameter, $a$ ) for the Nondispersed $\mathrm{H}_{2}$ Phases As Derived from SAXS

Investigations That Were Carried Out at $25^{\circ} \mathrm{C}$ on the Oil-Loaded Systems $(\alpha=19)$ for the Ternary MLO-TC-Water $(\beta=0)$ and the Quaternary MLO-DGMO-TC-Water $(\beta=100)$ Systems

\begin{tabular}{ccc}
\hline $\begin{array}{c}\text { investigated } \\
\text { nondispersed } \mathrm{H}_{2} \text { system }\end{array}$ & $\begin{array}{c}\text { water } \\
\text { content }\end{array}$ & $a[\mathrm{~nm}]$ \\
\hline MLO-TC-water & 10 & 4.50 \\
& 15 & 4.80 \\
& 20 & 5.90 \\
MLO-DGMO $(\beta=25)-\mathrm{TC}-$ water & $\mathbf{2 5}^{a}$ & $\mathbf{6 . 2 0}$ \\
& 10 & 5.31 \\
& 15 & 5.86 \\
& 20 & 6.54 \\
& 30 & 7.76 \\
& $\mathbf{3 5}^{a}$ & $\mathbf{8 . 6 0}$
\end{tabular}

${ }^{a}$ These samples represent SAXS data for both systems (the dispersed and the fully hydrated nondispersed $\mathrm{H}_{2}$ with excess water). They have the same structure parameters.

hexagonal or W/O microemulsion to a cubic phase. However, the increase in the lattice parameter due to the addition of DGMO is remarkable (see also Table 4). To understand the reason for this significant change in the internal nanostructures of these oil-loaded dispersions in the presence of DGMO, we decided to study the effect of partially replacing MLO $(\beta=0)$ by DGMO $(\beta=100)$ on the water solubilization capacity and the structure parameters of one of these oil-loaded nondispersed phases $(\alpha=$ 19). Upon increasing the DGMO content in the bulk phases from $\beta=0$ (Figure $7 \mathrm{a}$ ) to 100 (Figure $7 \mathrm{~b}$ ), the solubilized water content in the $\mathrm{H}_{2}$ phase significantly increases from approximately 25 to $35 \mathrm{wt} \%$ without any structural transition. In addition, the corresponding structure parameters, $a$, are much larger than those of the ternary MLO/TC/water systems at the same water content (see Table 4). These results show that the incorporation of DGMO with its bulky hydrophilic group into the interfacial area leads to the formation of $\mathrm{H}_{2}$ phases with larger hydrophilic (headgroups plus water) cylindrical channels. Therefore, DGMO alters the properties of the internal structure, offering a tool to induce the solubilization of hydrophilic additives and the entrapment of enzymes and proteins. Angelov et al. ${ }^{62}$ reported on the formation of $P n 3 m$ cubic phases with large water channels by the addition

(62) Angelov, B.; Angelova, A.; Ollivon, M.; Bourgaux, C.; Campitelli, A J. Am. Chem. Soc. 2003, 125, 7188-7189. 

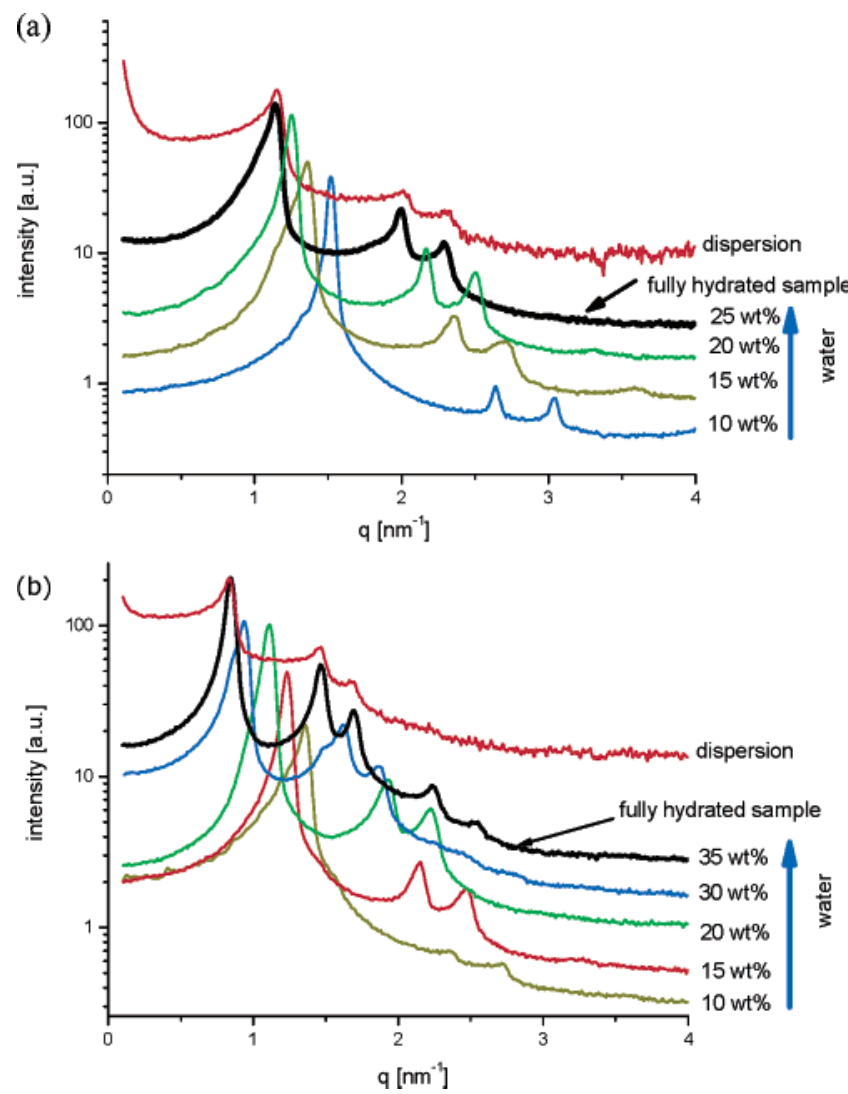

Figure 7. Comparison of the scattering curves from the same TCloaded hexosomes $(\alpha=19)$ with those from the nondispersed bulk $\mathrm{H}_{2}$ samples at $25^{\circ} \mathrm{C}$. The SAXS measurements were carried out with two systems having two different $\beta$ values: 0 (a) and 100 (b). The curves are shifted by a constant arbitrary factor for better visibility.

of octylglucoside to the binary $\mathrm{MO} /$ water system. In a recent review, Spicer ${ }^{40}$ described an approach to modify the bicontinuous cubic phases for loading additives, through functionalization, by incorporating amphiphilic materials into the liquid crystals. It is important to note that our data show a good structural agreement between the dispersed $\mathrm{H}_{2}$ phases and the fully hydrated bulk phases (see examples in Figure 7 and Table 4). This shows that F127 does not essentially influence the internal hexagonal structure, which confirms previous reports on the stabilization of hexosomes. ${ }^{28,32}$ However, we would like to mention that this does not imply that there is less F127 incorporated into these hexosomes than in the cubosomes discussed above. In our ongoing parallel study on the effect of fatty impurities on the internal structure of our dispersions, we found systems based on pure lipids in which we have observed that the same level of incorporated F127 has a much more pronounced effect on cubic phases than on hexagonal phases, but this is out of the scope of our present report, and, therefore, the data are not shown. We will discuss them in detail in our future report.

\section{Effect of Temperature on the Internal Structure of Oil-} Loaded MLO/DGMO-Based Aqueous Dispersions. Figure 8 shows the temperature dependence of scattering curves for two TC-loaded aqueous dispersions with the same $\alpha$ value of 6 but two different $\beta$ values: for MLO ( $\beta=0$, see Figure $8 \mathrm{a})$ and MLO/DGMO mixture $(\beta=50$, Figure $8 b)$. For MLO/TC dispersion, the temperature increase shifts the peaks to higher $q$ values, it reduces the structure parameters, and finally it induces changes in the symmetry from $\mathrm{H}_{2}$ to $\mathrm{L}_{2}$ of the confined nanostructures as shown in Figure 8a. In the presence of DGMO, as shown in Figure 8b, the formation of cubosomes instead of hexosomes with an internal structure of the symmetry $\operatorname{Im} 3 m$ at
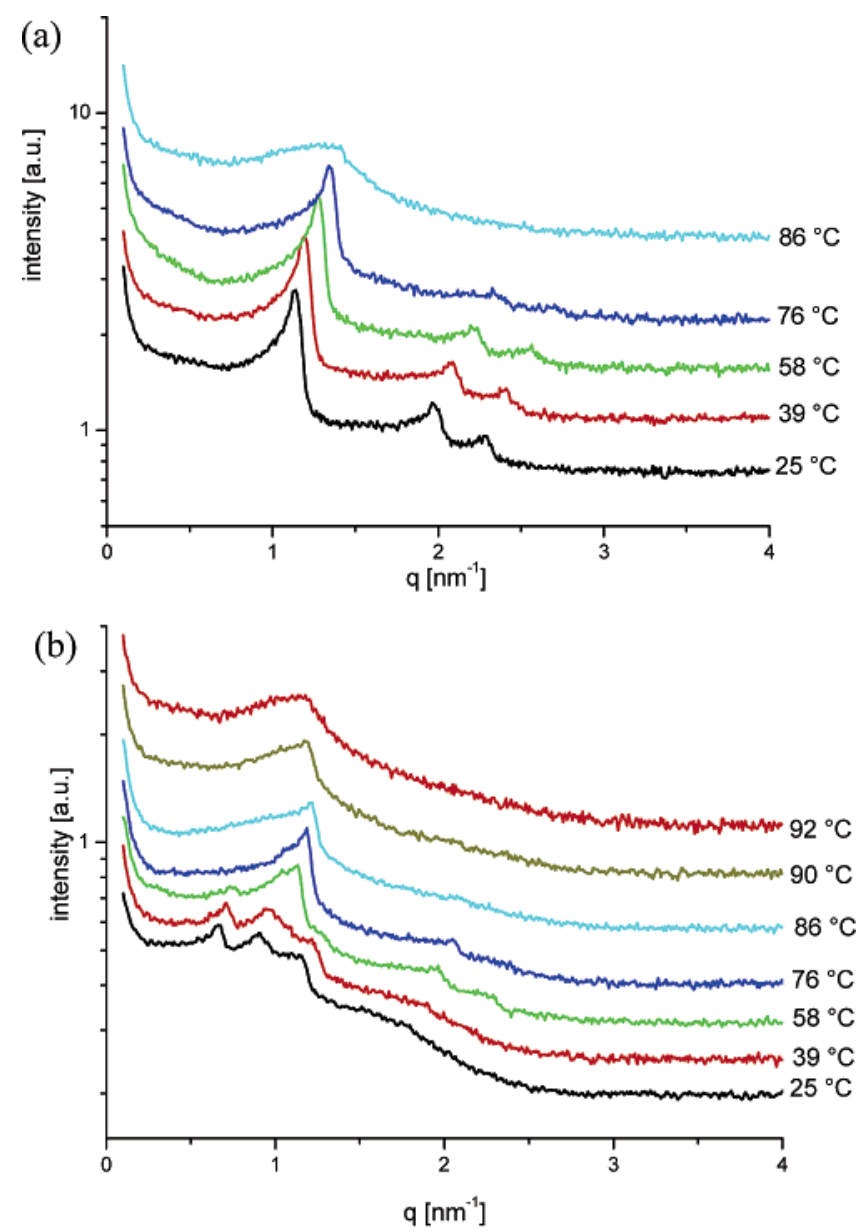

Figure 8. Temperature dependence of scattering curves for the MLO-based aqueous dispersions with the same solubilized oil content $(\alpha=6)$ but two different $\beta$ values of 0 (a) and 50 (b). The temperature effect was carried out in the range of $25-86^{\circ} \mathrm{C}$ for (a) and $25-92^{\circ} \mathrm{C}$ for (b). The curves are shifted by a constant arbitrary factor for better visibility.

low temperatures is promoted, and the temperature increase induces changes in the symmetry of the confined nanostructure from $\operatorname{Im} 3 m$ phase via $\mathrm{H}_{2}$ to $\mathrm{L}_{2}$ at high temperatures. It is also worth noting that DGMO significantly increases the temperature for all structural transitions. For instance, the $\mathrm{H}_{2} \rightarrow \mathrm{L}_{2}$ transition temperature of the dispersion with $\alpha=6$ increases from approximately 86 to $92{ }^{\circ} \mathrm{C}$ when MLO is replaced by MLO/ DGMO. This temperature is slightly higher than that reported in our recent work for the dispersed oil-free MLO-water system (the transition occurred at $87^{\circ} \mathrm{C}$ ). ${ }^{42}$ It indicates that the incorporation of the DGMO molecules into the interfacial area alters the flexibility of the interfacial film and makes the transition from $\mathrm{H}_{2}$ to $\mathrm{L}_{2}$ occur at higher temperatures.

5. Effect of Soybean Phosphatidylcholine (PC) on the Internal Structure of MLO-Based Aqueous Dispersions. This part describes some of our results on the effect of the partial replacement of MLO by the well-known balanced surfactant PC that favors the formation of $\mathrm{L}_{\alpha}$ phases. It is here that we present an approach similar to that for DGMO, modifying the MLObased phases by making the spontaneous curvature less negative. Figure 9a shows the structural transformations in the nondispersed MLO-PC mixture in the absence of oil (at $\alpha=0$ and $\beta=25$ ) with increasing water content: an inverse $\mathrm{L}_{2}$ phase is formed in the absence of water ( $0 \mathrm{wt} \%$ water), and the addition of water causes at $25^{\circ} \mathrm{C}$ a transition from $\mathrm{L}_{2} \rightarrow \mathrm{H}_{2}$ (5 to $15 \mathrm{wt} \%$ water) $\rightarrow \operatorname{Ia} 3 d$ (20 and 25 wt $\%$ water) $\rightarrow \operatorname{Pn3m}$ (the fully hydrated sample). At a high water content ( $\geq 30 \mathrm{wt} \%)$, the water 

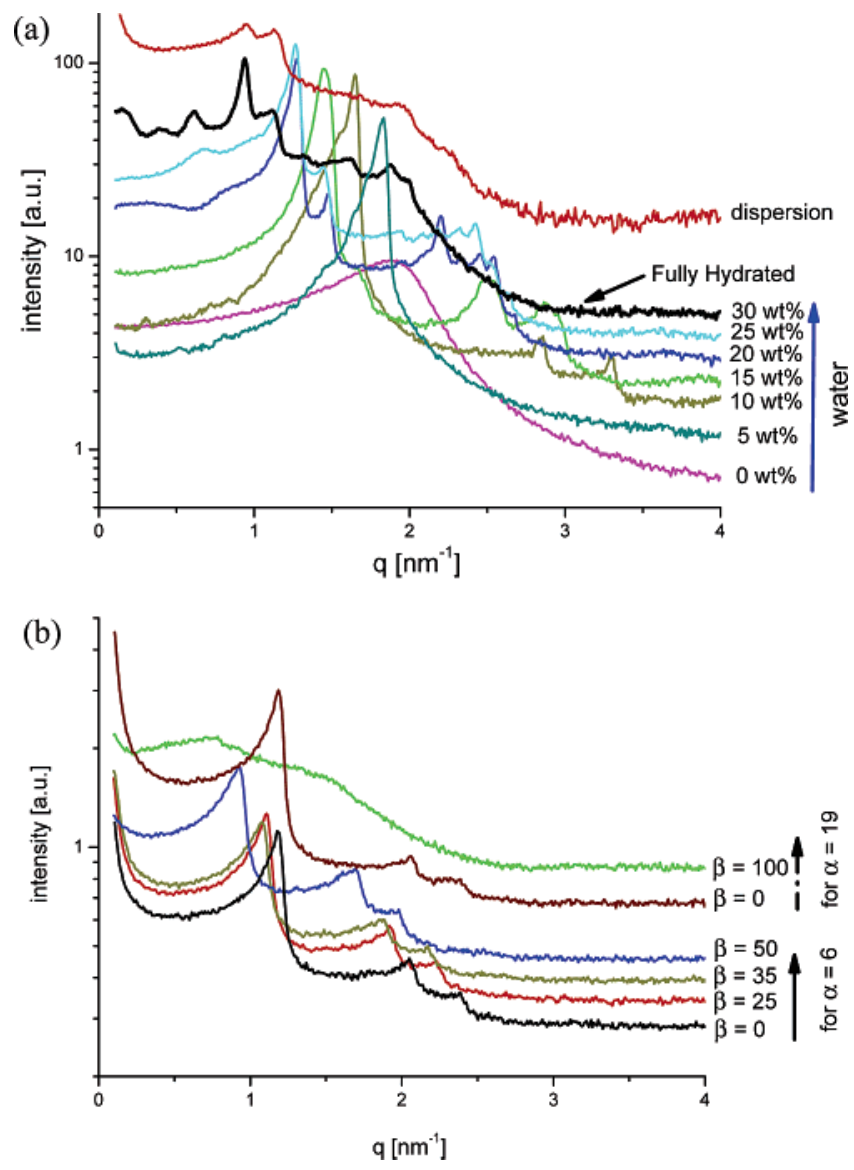

Figure 9. (a) Comparison of the scattering curve from the oil-free MLO-based dispersion $(\alpha=0, \beta=25$, red line) with those from the oil-free nondispersed MLO/PC bulk samples at $\beta=25$ with different water content at $25^{\circ} \mathrm{C}$. (b) Effect of PC content on the scattering curves of TC-loaded aqueous dispersions at $25^{\circ} \mathrm{C}$ for two dispersions having $\alpha$ ratios of 6 and 19, respectively, and with different $\beta$ values in the range of $0-50$ for the dispersion with $\alpha$ ratios of 6 , and two different $\beta$ values ( 0 and 100) for the dispersion with $\alpha$ ratios of 19 . The intensities were shifted by a constant arbitrary factor for better visibility.

solubilization capacity of this sample is exceeded and the $P n 3 m$ phase is in equilibrium with excess water. Above this fully hydrated condition, no more change is observed in the peak positions and in the corresponding structure parameters (the mean lattice parameters, $a$, for these phases are presented in Table 2). As shown in Figure 9, the internal structure is preserved after dispersing the MLO/PC-based cubic phase with excess water as verified by SAXS measurements. The scattering curve of the aqueous dispersion shows a cubic structure of the type $P n 3 m$, possibly a mixture with $\operatorname{Im} 3 \mathrm{~m}$ (cubosomes), and it corresponds to that of the nondispersed fully hydrated bulk phase with the same $\beta$ value, but with a much lower degree of order. The fragmentation of the cubic phases into submicrometer-sized particles only leads to a decrease in the sharpness of the peaks of the scattering curve in the dispersed sample, and it makes the peaks weaker. This result also implies that the polymer that is used to stabilize the particles does a good job as an efficient stabilizer: it adsorbs to the outer surface of the fragmented cubic phase and does not incorporate into the internal structure in contrast to what is observed for the dispersed MLO/DGMO cubic phases. This means that the incorporation of the polymer into the lipid bilayers is easier in the MLO/DGMO mixture than that in a MLO/PC system.

The aqueous dispersions of the ternary MLO/PC/TC with different $\beta$ values (in the range of $0-50$ ) and a fixed $\alpha$ value of 6 were investigated by SAXS at $25^{\circ} \mathrm{C}$ to identify their internal nanostructures (see Figure 9b). PC shifts the observed peaks in the scattering curves for the hexosomes to lower angles, corresponding to larger structures similar to that observed with TC-loaded MLO/DGMO-based dispersions, but without promoting any structural transitions. Both DGMO and PC alter the internal structure properties. However, the results reveal that DGMO is more effective for tuning back the internal confined structure back from $\mathrm{H}_{2}$ to cubic phase, when we compare the scattering curves for the dispersions containing the same amount of solubilized TC (Figure 5 vs Figure 9b). In addition, we found that, for dispersions with a higher amount of solubilized TC content $(\alpha=19)$, replacement of MLO by a mixture of MLO/PC ( $\beta=100$, Figure 9b) makes the internal nanostructure less ordered: the peaks are very broad, and it is a difficult task to determine the internal structure in these dispersions.

\section{Conclusions}

The present work deals with an important topic in nanotechnology, how to effectively control the nanostructure in a confined geometry. It proves that the internal structures of emulsified particles based on MLO-water binary and ternary MLO-TCwater mixtures are modulated by varying the lipid composition. We found that the replacement of MLO by the binary MLO/ DGMO or MLO/PC blends significantly affects the internal nanostructures of the kinetically stabilized aqueous dispersions. Our results show that DGMO has a counter effect to that of TC and induces a reversed transition from hexosomes to cubosomes for the TC-loaded dispersions. However, TC has a higher impact on the confined nanostructures than does DGMO.

It is of special interest from an applicational point of view that DGMO molecules alter the properties of the confined nanostructures of our aqueous dispersions; they strongly increase the water solubilization capacity of such phases and thereby increase the size of their hydrophilic domains. This offers excellent media for the solubilization and entrapment of various hydrophilic active molecules, enzymes, or proteins. It is interesting as a useful approach for the formation of novel products. Our results also reveal that PC molecules similar to those of DGMO affect the properties of the internal nanostructure. However, PC leads to the formation of fragmented dispersed particles with a lower degree of order.

LA061303V 